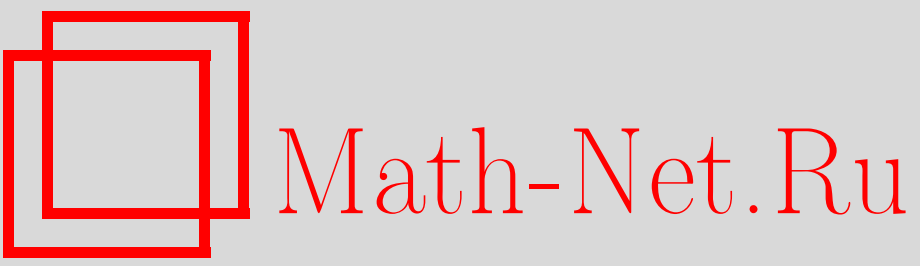

С. Н. Сторчак, Реономные однородные точечные преобразования и репараметризация путей в континуальных интегралах по квазимерам, ТМФ, 1997, том 111, номер 2, 234-241

DOI: https://doi.org/10.4213/tmf1003

Использование Общероссийского математического портала Math-Net.Ru подразумевает, что вы прочитали и согласны с пользовательским соглашением

http: //www . mathnet.ru/rus/agreement

Параметры загрузки:

IP: 18.207 .199 .55

26 апреля 2023 г., 10:23:40 
ТЕОРЕТИЧЕСКАЯ

И МАТЕМАТИЧЕСКАЯ

ФИЗИКА

Том 111, № 2

май, 1997

\title{
С.Н. Сторчак* \\ РЕОНОМНЫЕ ОДНОРОДНЫЕ ТОЧЕЧНЫЕ ПРЕОБРАЗОВАНИЯ И РЕПАРАМЕТРИЗАЦИЯ ПУТЕЙ В КОНТИНУАЛЬНЫХ ИНТЕГРАЛАХ ПО КВАЗИМЕРАМ
}

\begin{abstract}
Рассмотрены реономные однородные точечные преобразования и преобразования репараметризации путей в континуальных интегралах по квазимерам для линейных параболических дифференциальных уравнений четвертого поря дка с явной зависимостью коэффициентов от времени. Найдено интегральное соотношение между функциями Грина для дифференциальных уравнений четвертого порядка, находящихся в соответствии друг с другом. Дифференциальный оператор преобразованного уравнения в частном случае совпадает с конформно-ковариантным оператором Бола, хорошо известным в точно интегрируемых и конформных моделях.
\end{abstract}

\section{1. ВВЕДЕНИЕ}

Данная статья продолжает исследование континуальных интегралов по квазимерам, начатое в работах $[1,2]$. Такие интегралы, определяемые как слабьй предел последовательности конечнократных распределений, являются обобщениями винеровских континуальных интегралов и могут быть использованы для нахождения решений линейных параболических уравнений высших порядков.

По аналогии с обычным стохастическим исчислением для континуальных интегралов по квазимерам можно ввести [3-6] понятие стохастического интеграла, лемму Ито о дифференцировании сложной функции, формулу Фейнмана-Каца и т.д. Развитое "стохастическое исчисление" основано на процессах, связанных с параболическими уравнениями с высшими производными. Хотя эти процессы и не являются случайными в общепринятом вероятностном смысле, но они в этих работах также были названы случайными $[4,6]$. Мы, как и прежде, будем следовать этой терминологии.

Целью работы является исследование преобразований континуальных интегралов при изменении параметризации "путей” (репараметризации или “замене времени" [7]) и при реономных однородных точечных преобразованиях переменных интегрирования ("преобразованиях пространства"). Мы распространяем предложенную ранее схему исследования континуальных интегралов по квазимерам, основанную на "стохастическом исчислении", на континуальные интегралы для параболических дифференциальных

\footnotetext{
${ }^{*}$ Институт физики высоких энергий, Протвино, Московская область, Россия
} 
уравнений четвертого порядка с явной зависимостью коэффициентов от времени. Согласно этой схеме правила преобразования континуальных интегралов можно вывести, если рассмотреть преобразования случайных процессов, поскольку последние определяют квазимеры в континуальных интегралах. Именно с такой точки зрения в работе и будут рассматриваться преобразования континуальных интегралов.

\section{2. ОПРЕДЕЛЕНИЯ}

Нашим исходным уравнением будет следуюшее дифференциальное уравнение:

$$
\begin{aligned}
& \left(\frac{\partial}{\partial t_{a}}+\widehat{H}_{\kappa}\left(x_{a}\right)\right) \psi_{t_{b}}\left(x_{a}, t_{a}\right)=0, \\
& \psi_{t_{b}}\left(x, t_{b}\right)=\chi_{0}(x) \quad\left(t_{b}>t_{a}\right),
\end{aligned}
$$

где

$$
\widehat{H}_{\kappa}\left(x_{a}\right)=(-1) \widetilde{m}\left(\frac{f_{4}}{4 !} \frac{\partial^{4}}{\partial x_{a}^{4}}+\frac{f_{3}}{3 !} \frac{\partial^{3}}{\partial x_{a}^{3}}+\frac{f_{2}}{2} \frac{\partial^{2}}{\partial x_{a}^{2}}+f_{1} \frac{\partial}{\partial x_{a}}\right)+V .
$$

Таким образом, в данном (нестационарном) случае мы рассматриваем обратное уравнение, т.е. уравнение по переменным $\left(x_{a}, t_{a}\right)$. По своему виду уравнение $(1)$ совпадает с исходным уравнением работы [1], т.е. коэффициенты $f_{i}$ и $V$ выражаются через переменные $a, b, c, d$ и их производные по тем же формулам, что и в работе [1] (формулы $(2),(3))$. Отличие состоит в том, что теперь вместо $\beta$ и $\delta$ в них стоят $\beta_{1}=-\beta$ и $\delta_{1}=-\delta$. При переходе к прямому уравнению, т.е. уравнению по переменным $\left(x_{b}, t_{b}\right), \beta_{1}$ и $\delta_{1}$ заменяются на $\beta$ и $\delta$.

Заметим, что если коэффициенты дифференциального уравнения являются достаточно гладкими функциями (что в работе и предполагается), то фундаментальное решение должно удовлетворять прямому уравнению (уравнению по $x_{b}, t_{b}$ ), но уже с сопряженным оператором.

Чтобы получить уравнение шредингеровского вида, нужно в прямом уравнении положить $\kappa=i$. Тогда оператор Гамильтона будет иметь вид

$$
\begin{aligned}
\widehat{H}= & \frac{1}{4 !\left(m_{4}\right)^{3}}(\sqrt{a} \widehat{p} \sqrt{a})^{4}+\frac{1}{3 !\left(m_{3}\right)^{2}}(\sqrt{b} \widehat{p} \sqrt{b})^{3}+ \\
& +\frac{1}{2 m_{2}}(\sqrt{c} \widehat{p} \sqrt{c})^{2}+(\sqrt{d} \widehat{p} \sqrt{d})+V_{0} .
\end{aligned}
$$

Этот оператор формально самосопряжен в пространстве функций со скалярным произведением $(\psi, \psi)=\int|\psi(x, t)|^{2} d x$ и соответствует классическому гамильтониану

$$
H=\frac{1}{4 !\left(m_{4}\right)^{3}} a^{4} p^{4}+\frac{1}{3 !\left(m_{3}\right)^{2}} b^{3} p^{3}+\frac{1}{2 m_{2}} c^{2} p^{2}+d p+V_{0} .
$$

Если распространить формулу Фейнмана-Каца из [6] на нестационарный случай, воспользовавшись методами, развитыми в [8] для обычных процессов, то решение уравнения (1) можно представить в виде

$$
\psi_{t_{b}}\left(x_{a}, t_{a}\right)=\int_{\Omega_{-}} d \mu^{n}(\omega) \exp \left(\int_{t_{a}}^{t_{b}} V(\eta(u), u) d u\right) \chi_{0}\left(\eta\left(t_{b}\right)\right)
$$


где $\Omega_{-}-$пространство таких путей $\omega$, что $\eta(t)=x_{a}+\omega(t), \omega\left(t_{a}\right)=0$. Представление (4) есть в действительности символическая запись слабого предела соответствующих дискретных аппроксимаций, подобных тем, что использовались в [6].

В основе доказательства формулы Фейнмана-Каца для уравнений с высшими производными лежит аналог известной формулы Ито о дифференцировании сложной функции $[4,6]$. Мы будем использовать формулу Ито в предположении сушествования решения стохастического дифференциального уравнения общего вида:

$$
x(t)=x\left(t_{a}\right)+\int_{t_{a}}^{t} A(u) d z^{1}(u)+\int_{t_{a}}^{t} B(u) d z^{2}(u)+\int_{t_{a}}^{t} C(u) d z^{3}(u)+\int_{t_{a}}^{t} D(u) d u,
$$

$d x=A d z^{1}+B d z^{2}+C d z^{3}+D d t, d z^{j}(j=1,2,3)$ - независимые стохастические дифференциалы и $d z^{4}=(-1) \widetilde{m} d t ; A \equiv A(x(u), u$ ) (и аналогично $B, C, D)$.

В нашем случае формула Ито (в дифференциальном виде) выглядит следуюшим образом:

$$
\begin{aligned}
d f(x(t), t)= & A f^{\prime} d z^{1}+\left[f^{\prime} B+\frac{1}{2} f^{\prime \prime} A^{2}\right] d z^{2}+\left[f^{\prime} C+f^{\prime \prime} A B+\frac{1}{3 !} f^{\prime \prime \prime} A^{3}\right] d z^{3}+ \\
& +(-1) \widetilde{m}\left[-\frac{1}{\widetilde{m}} \frac{\partial f}{\partial t}-\frac{f^{\prime} D}{\widetilde{m}}+\frac{1}{2} f^{\prime \prime}\left(2 A C+B^{2}\right)+\frac{1}{2} f^{\prime \prime \prime} A^{2} B+\frac{1}{4 !} f^{\mathrm{IV}} A^{4}\right] d t
\end{aligned}
$$

Будем предполагать, что случайный процесс $\eta$, порождающий квазимеру $\eta^{\mu}$ в (4), определяется из решения стохастического дифференциального уравнения следующего вида:

$$
d \eta(t)=f_{4}^{1 / 4} d z^{1}+\frac{1}{3} f_{3} f_{4}^{-1 / 2} d z^{2}+\frac{1}{2}\left(f_{2}-\frac{1}{9} f_{3}^{2} f_{4}^{-1}\right) f_{4}^{-1 / 4} d z^{3}-\widetilde{m} f_{1} d t .
$$

Выбор такого специального стохастического уравнения для определения процесса $\eta$ (и меры $\eta^{\mu}$ ) обусловлен необходимостью иметь для производящего генератора полугруппы (4) выражение, совпадаюшее с дифференциальным оператором уравнения (1). Заметим, что в нестационарном случае формула для производяшего генератора полугруппы обшего вида, т.е. содержашая экспоненту вместе со стохастическими интегралами по процессам $z^{1}, z^{2}, z^{3}$, совпадает с формулой (12) из [1].

Переходя в (4) к интегрированию по пространству “путей" с фиксированными начальными и конечными точками (в этом случае $\chi_{0}(x)=\delta\left(x-x_{b}\right)$ ), мы получим представление для плотности вероятности перехода $G_{\kappa}$ через континуальньй интеграл.

\section{3. РЕОНОМНЫЕ ОДНОРОДНЫЕ ТОЧЕЧНЫЕ ПРЕОБРАЗОВАНИЯ}

В классической механике реономное однородное точечное преобразование задается следуюшими формулами:

$$
x=f(y, t), \quad p=(d f / d y)^{-1} \tilde{p}
$$

В нашей предыдушей работе [9] было показано, как такие преобразования реализуются в обычных континуальных интегралах. 
Формула для замены переменной интегрирования $x=f(y, t)$ в континуальном интеграле (4) может быть получена двумя эквивалентными способами. Первый способ это непосредственное преобразование плотности вероятности распределения (cм. $[1,9])$. Второй способ связан с преобразованием континуального интеграла (4) при переходе от случайного процесса $\eta(t)$, определенного в $(5)$, к процессу $\xi(t)$, стохастическое дифференциальное уравнение которого есть

$$
\begin{aligned}
& d \xi=A_{\xi} d z^{1}+B_{\xi} d z^{2}+C_{\xi} d z^{3}+D_{\xi} d t \\
& A_{\xi}=\frac{1}{f^{\prime}} f_{4}^{1 / 4}, \quad B_{\xi}=\frac{1}{3} \frac{1}{f^{\prime}} f_{3} f_{4}^{-1 / 2}-\frac{2}{3} \frac{f^{\prime \prime}}{f^{\prime 3}} f_{4}^{1 / 2}, \\
& C_{\xi}=\frac{1}{2} \frac{1}{f^{\prime}}\left(f_{2}-\frac{1}{9} f_{3}^{2} f_{4}^{-1}\right) f_{4}^{-1 / 4}-\frac{1}{6}\left(\frac{7}{4} \frac{f^{\prime \prime \prime}}{f^{\prime 4}}-\frac{139}{24} \frac{f^{\prime \prime 2}}{f^{\prime 5}}\right) f_{4}^{3 / 4}-\frac{19}{36} \frac{f^{\prime \prime}}{f^{\prime 3}} f_{4}^{-1 / 4} f_{3}, \\
& D_{\xi}=\tilde{m}\left[\left(\frac{1}{8} \frac{f^{\mathrm{IV}}}{f^{\prime 5}}-\frac{4}{3} \frac{f^{\prime \prime \prime} f^{\prime \prime}}{f^{\prime 6}}+\frac{17}{8} \frac{f^{\prime \prime 3}}{f^{\prime 7}}\right) f_{4}+\left(\frac{5}{12} \frac{f^{\prime \prime \prime}}{f^{\prime 4}}-\frac{11}{8} \frac{f^{\prime \prime 2}}{f^{\prime 5}}\right) f_{3}+\frac{f^{\prime \prime}}{f^{\prime 3}} f_{2}-\frac{1}{f^{\prime}} f_{1}\right] .
\end{aligned}
$$

Переход от $\eta$ в $\xi$ происходит в два этапа. Сначала процесс $\eta$ преобразуется в $\xi_{1}$ : $\eta(t)=f\left(\xi_{1}(t), t\right)$ (стохастическое дифференциальное уравнение для $\xi_{1}$ выводится при помоши формулы Ито). А затем $\xi_{1}$ преобразуется в $\xi$. Первое преобразование есть аналог фазовых преобразований обычных случайных процессов. Оно приводит к изменению нормировки волновых функций. Поэтому мы должны сделать еше одно преобразование: от процесса $\xi_{1}$ к процессу $\xi$. Такое преобразование в континуальном интеграле по квазимере выполняется в соответствии с формулой Гирсанова-Камерона-Мартина (см. [1]).

Результат этих преобразований удобно представить через преобразование символов континуальных интегралов. Тогда реономное однородное точечное преобразование континуального интеграла по квазимере, переписанное через преобразование символов континуальных интегралов, будет выглядеть таким же образом, как и в работе [1] (формула (16)). Но теперь гамильтониан $\widetilde{H}_{1}$ из правой части формулы равен

$$
\begin{aligned}
\widetilde{H}_{1}= & \frac{1}{4 !\left(m_{4}\right)^{3}} a^{4} f^{\prime-4} \tilde{p}^{4}+\frac{1}{3 !\left(m_{3}\right)^{2}} b^{3} f^{\prime-3} \tilde{p}^{3}+\frac{1}{2 m_{2}} c^{2} f^{\prime-2} \tilde{p}^{2}+ \\
& +d f^{\prime-1} \tilde{p}-\left(\frac{\partial f(y, t)}{\partial t}\right) f^{\prime-1} \tilde{p}+V_{0}
\end{aligned}
$$

где $a \equiv a(F(y, t), t)$ (и аналогично $b, c, d$ и $\left.V_{0}\right)$.

Для гамильтонианов $\widetilde{H}_{1}$ и $H$ используется правило квантования (2). Связь между оператором Гамильтона $\widehat{H}_{1}$ для классического гамильтониана $(7)$ и производящим генератором $H_{\kappa}$ полугруппы, полученной в результате перехода от процесса $\eta$ к процессу $\xi$, определяется формулой $\widehat{H}_{1}=-\left.\frac{\hbar}{\kappa} \widehat{H}_{\kappa}(y)\right|_{\kappa=i}$, где $\widehat{H}_{\kappa}(y)$ есть

$$
\begin{aligned}
& (-1) \widetilde{m}\left\{\frac{1}{4 !} \frac{1}{f^{\prime 4}} f_{4} \frac{d^{4}}{d y^{4}}+\frac{1}{3 !}\left[\frac{1}{f^{\prime 3}} f_{3}-2 \frac{f^{\prime \prime}}{f^{\prime 5}} f_{4}\right] \frac{d^{3}}{d y^{3}}+\right. \\
& +\frac{1}{2}\left[\frac{1}{f^{\prime 2}} f^{2}-\frac{3}{2} \frac{f^{\prime \prime}}{f^{\prime 4}} f_{3}+\left(\frac{19}{8} \frac{f^{\prime \prime 2}}{f^{\prime 6}}-\frac{7}{12} \frac{f^{\prime \prime \prime}}{f^{\prime 5}}\right) f_{4}\right] \frac{d^{2}}{d y^{2}}+
\end{aligned}
$$




$$
\begin{aligned}
& +\left[-\frac{1}{\widetilde{m}} \frac{\partial f}{\partial t} \frac{1}{f^{\prime}}+\frac{1}{f^{\prime}} f_{1}-\frac{f^{\prime \prime}}{f^{\prime 3}} f_{2}+\left(-\frac{5}{12} \frac{f^{\prime \prime \prime}}{f^{\prime 4}}+\frac{11}{8} \frac{f^{\prime \prime 2}}{f^{\prime 5}}\right) f_{3}+\right. \\
& \left.+\left(-\frac{1}{8} \frac{f^{\mathrm{IV}}}{f^{\prime 5}}+\frac{4}{3} \frac{f^{\prime \prime \prime} f^{\prime \prime}}{f^{\prime 6}}-\frac{17}{8} \frac{f^{\prime \prime 3}}{f^{\prime 7}}\right) f_{4}\right] \frac{d}{d y}+ \\
& +\left[-\frac{1}{2 \widetilde{m}} \frac{\partial^{2} f}{\partial y \partial t} \frac{1}{f^{\prime}}+\frac{1}{2 \widetilde{m}} \frac{\partial f}{\partial t} \frac{f^{\prime \prime}}{f^{\prime 2}}-\frac{V}{\widetilde{m}}-\frac{1}{2} \frac{f^{\prime \prime}}{f^{\prime 2}} f_{1}+\right. \\
& +\left(-\frac{1}{48} \frac{f^{\mathrm{V}}}{f^{\prime 5}}+\frac{13}{48} \frac{f^{\mathrm{IV}} f^{\prime \prime}}{f^{\prime 6}}+\frac{17}{96} \frac{f^{\prime \prime \prime 2}}{f^{\prime 6}}-\frac{161}{96} \frac{f^{\prime \prime \prime} f^{\prime \prime 2}}{f^{\prime 7}}+\frac{195}{128} \frac{f^{\prime \prime 4}}{f^{\prime 8}}\right) f_{4}+ \\
& \left.\left.+\left(-\frac{1}{12} \frac{f^{\mathrm{IV}}}{f^{\prime 4}}+\frac{17}{24} \frac{f^{\prime \prime \prime} f^{\prime \prime}}{f^{\prime 5}}-\frac{f^{\prime \prime 3}}{f^{\prime 6}}\right) f_{3}+\left(-\frac{1}{4} \frac{f^{\prime \prime \prime}}{f^{\prime 3}}+\frac{5}{8} \frac{f^{\prime \prime 2}}{f^{\prime 4}}\right) f_{2}\right]\right\} \text {. }
\end{aligned}
$$

\section{4. РЕПАРАМЕТРИЗАЦИЯ ПУТЕЙ В КОНТИНУАЛЬНЫХ ИНТЕГРАЛАХ}

В основе подхода к преобразованию континуальных интегралов по квазимерам при изменении параметризации путей лежит процедура случайной замены времени в процессах, порождающих квазимеры. Согласно этой процедуре в преобразуемом процессе переменная $t$ заменяется на случайную функцию $\tau_{t}$. В результате получается новый случайный процесс. В нашем случае мы имеем $\eta(t) \equiv \eta(t, \omega)=\xi\left(\tau_{t}(\omega), \omega\right)$. Случайную функцию $\tau_{t}$ будем находить из решения следуюшего интегрального уравнения для функции $\varphi(s)$, обратной к $\tau_{t}$ :

$$
\varphi(s)=t_{a}+\int_{s_{a}}^{s} e^{-4 \sigma(\xi(u), \varphi(u))} d u
$$

Заметим, что, как и в обычных процессах [8], случайная замена времени в неоднородных по времени процессах, связанных с квазимерами, получается путем распространения понятия стохастического интеграла со случайными пределами (марковскими моментами) на интегралы от функций, явно зависящих от времени.

Для преобразования континуального интеграла перейдем сначала от полугруппы (4) к ее резольвенте

$$
\left(R_{\lambda} \xi_{0}\right)\left(x_{a}, t_{a}\right)=\int_{t_{a}}^{\infty} e^{-\lambda\left(t_{b}-t_{a}\right)} d t_{b} \int_{\Omega_{-}} d \mu^{\eta} \xi_{0}\left(\eta\left(t_{b}\right)\right) \exp \left(\int_{t_{a}}^{t_{b}} V(\eta(u), u) d u\right),
$$

а затем в резольвенте произведем случайную замену времени. Эти преобразования аналогичны тем, которые были сделаны в предыдущих работах $[1,2]$.

После выполненных преобразований в резольвенте мы вновь сталкиваемся с нежелательным изменением нормировки волновых функций. Исправляя этот недостаток, мы вынуждены сделать еще одно дополнительное преобразование, которое переводит процесс $\xi$ в $\widetilde{\xi}$ и меру $\eta^{\xi}$ - в $\eta^{\widetilde{\xi}}$. Якобиан этого преобразования вычисляется при помоши формулы Гирсанова-Камерона-Мартина (см. [1]). Мы не будем приводить здесь подробных выкладок (их можно найти в [1]), а сразу запишем результат. Отметим только, что при 
выводе тождества Ито, которое помогает избавиться от стохастического интеграла по $z^{1}$, должна применяться следуюшая формула ${ }^{1)}$ для коэффициента $K_{1}$ :

$$
K_{1}=\Lambda_{1}+\tilde{m}\left(\Lambda_{2} \Lambda_{4}+\frac{1}{2} \Lambda_{3}^{2}-\Lambda_{3} \Lambda_{4}^{2}+\frac{1}{4} \Lambda_{4}^{4}\right)+\frac{\partial \sigma}{\partial s} .
$$

Для $K_{4}, K_{3}, K_{2}$ формулы остаются такими же, как и в работе [1].

После обратного преобразования резольвенты придем к окончательной формуле для преобразования континуального интеграла при репараметризации путей:

$$
\begin{aligned}
\left(G \chi_{0}\right)\left(x_{a}, t_{a} ; t_{b}\right)= & \frac{1}{2 \pi \hbar} \int_{-\infty}^{\infty} e^{-\frac{i}{\hbar} E\left(t_{b}-t_{a}\right)} d E \int_{s_{a}}^{\infty} d s_{b} \int_{\Omega_{-}} d \mu^{\widetilde{\xi}} \chi_{0}\left(\widetilde{\xi}\left(s_{b}\right)\right) \times \\
& \times e^{-2 \sigma\left(\widetilde{\xi}\left(s_{a}\right), \varphi\left(s_{a}\right)\right)} e^{-2 \sigma\left(\widetilde{\xi}\left(s_{b}\right), \varphi\left(s_{b}\right)\right)} \exp \left(\frac{i}{\hbar} E \int_{s_{a}}^{s_{b}} e^{-4 \sigma} d s\right) \times \\
& \times \exp \left\{\int_{s_{a}}^{s_{b}}\left[J_{3} d \tilde{z}^{2}(s)+J_{2} d \tilde{z}^{3}(s)+\left(J_{1}+e^{-4 \sigma} V\right)\right] d s\right\},
\end{aligned}
$$

где $J_{i}$ - функции от $\sigma, f_{i}$ и их производных, которые зависят от $(\widetilde{\xi}(s), \varphi(s))$, и $\widetilde{\xi}\left(s_{a}\right)=$ $x_{a}, \widetilde{\xi}\left(s_{b}\right)=x_{b}$. Производяшим оператором для правой части формулы (10) является оператор

$$
\begin{aligned}
& -\widetilde{m} e^{-4 \sigma}\left\{\frac{1}{4 !} f_{4} \frac{d^{4}}{d x^{4}}+\frac{1}{3 !}\left[f_{3}-2 \sigma^{\prime} f_{4}\right] \frac{d^{3}}{d x^{3}}+\frac{1}{2}\left[f_{2}-2 \sigma^{\prime} f_{3}+\epsilon f_{4}\right] \frac{d^{2}}{d x^{2}}+\right. \\
& +\left[f_{1}-2 \sigma^{\prime} f_{2}+\epsilon f_{3}+\left(-\frac{1}{3} \sigma^{\prime \prime \prime}+2 \sigma^{\prime \prime} \sigma^{\prime \prime}-\frac{4}{3} \sigma^{3}\right) f_{4}\right] \frac{d}{d x}+ \\
& +\left[-2 \sigma^{\prime} f_{1}+\epsilon f_{2}+\left(-\frac{1}{3} \sigma^{\prime \prime \prime}+2 \sigma^{\prime \prime} \sigma^{\prime \prime}-\frac{4}{3} \sigma^{\prime 3}\right) f_{3}+\frac{2}{\widetilde{m}} \frac{\partial \sigma}{\partial s} e^{4 \sigma}+\right. \\
& \left.\left.+\left(-\frac{1}{12} \sigma^{\mathrm{IV}}+\frac{2}{3} \sigma^{\prime \prime \prime} \sigma^{\prime \prime}+\frac{1}{2} \sigma^{\prime \prime 2}-2 \sigma^{\prime \prime} \sigma^{\prime 2}+\frac{2}{3} \sigma^{\prime 4}\right) f_{4}-\frac{V}{\widetilde{m}}-\frac{i}{\hbar \widetilde{m}} E\right]\right\}
\end{aligned}
$$

Здесь $\epsilon=\left(2 \sigma^{\prime 2}-\sigma^{\prime \prime}\right)$ и все функции уже зависят от $(x, t)$.

Теперь можно в континуальном интеграле по квазимере выполнить последовательно два преобразования: реономное однородное точечное преобразование (6) и преобразование репараметризации путей (8) со специальным выбором функции $\sigma: d t / d s=$ $(d f / d y)^{4}$. Эти преобразования приводят к следующему интегральному соотношению меж ду функциями Грина:

$$
\begin{aligned}
G_{\kappa}\left(x_{b}, t_{b} ; x_{a}, t_{a}\right)= & \int d y_{a} \delta\left(y_{a}-g\left(x_{a}, t_{a}\right)\right) \int d y_{b} \delta\left(y_{b}-g\left(x_{b}, t_{b}\right)\right) \times \\
& \times\left|\frac{\partial f}{\partial y}\right|^{3 / 2}\left(y_{a}, t_{a}\right)\left|\frac{\partial f}{\partial y}\right|^{3 / 2}\left(y_{b}, t_{b}\right) \times \\
& \times \frac{1}{2 \pi \hbar} \int_{-\infty}^{\infty} e^{-\frac{i}{\hbar} E\left(t_{b}-t_{a}\right)} d E \int_{s_{a}}^{\infty} d s_{b} \widetilde{G}_{\kappa}\left(y_{a}, s_{a} ; y_{b}, s_{b}\right) .
\end{aligned}
$$

\footnotetext{
1) В [1] по ошибке было дано неправильное выражение для коэффициента $K_{1}$. Правильное выражение, которое фактически и использовалось в [1] для вычисления якобианов $J_{i}$, совпадает с (9).
} 
Здесь $g$ есть обратная функция к $f$ по переменной $x, G_{\kappa}$ - функция Грина (фундаментальное решение) исходного уравнения $(1)$, а $\widetilde{G}_{\kappa}-$ функция Грина аналогичного уравнения (но в переменных $s_{a}$ и $y_{a}$ ) с дифференциальным оператором $\widehat{\widetilde{H}}_{\kappa}\left(y_{a}\right)$, равным

$$
\begin{aligned}
(-1) & \widetilde{m}\left\{\frac{1}{4 !} f_{4} \frac{d^{4}}{d y_{a}^{4}}+\frac{1}{3 !} f^{\prime} f_{3} \frac{d^{3}}{d y_{a}^{3}}+\frac{1}{2}\left[f^{\prime 2} f_{2}+\frac{1}{2} f^{\prime \prime} f_{3}+\right.\right. \\
+ & \left.\frac{5}{12}\left(\frac{f^{\prime \prime \prime}}{f^{\prime}}-\frac{3}{2}\left(\frac{f^{\prime \prime}}{f^{\prime}}\right)^{2}\right) f_{4}\right] \frac{d^{2}}{d y_{a}^{2}}+\left[-\frac{1}{\widetilde{m}} f^{\prime 3} \frac{\partial f}{\partial t}+f^{\prime 3} f_{1}+f^{\prime \prime} f^{\prime} f_{2}+\right. \\
& \left.+\left(\frac{7}{12} f^{\prime \prime \prime}-\frac{5}{8} \frac{f^{\prime \prime 2}}{f^{\prime}}\right) f_{3}+\frac{5}{24}\left(\frac{f^{\mathrm{IV}}}{f^{\prime}}-4 \frac{f^{\prime \prime \prime} f^{\prime \prime}}{f^{\prime 2}}+3\left(\frac{f^{\prime \prime}}{f^{\prime}}\right)^{3}\right) f_{4}\right] \frac{d}{d y_{a}}- \\
& -\frac{1}{\widetilde{m}}\left(\frac{1}{2} f^{\prime 3} \frac{\partial^{2} f}{\partial t \partial y}+\frac{3}{2} f^{\prime \prime} f^{\prime 2} \frac{\partial f}{\partial t}+\frac{\partial}{\partial s} \ln \left(\frac{\partial f}{\partial y}\right)^{2}+f^{\prime 4} V+\frac{i}{\hbar} f^{\prime 4} E\right)+ \\
& \left.+\frac{5}{16}\left(\frac{1}{5} \frac{f^{\mathrm{V}}}{f^{\prime}}-\frac{f^{\mathrm{IV}} f^{\prime \prime}}{f^{\prime 2}}-\frac{1}{2} \frac{f^{\prime \prime \prime} 2}{f^{\prime 2}}+\frac{5}{2} \frac{f^{\prime \prime \prime} f^{\prime \prime 2}}{f^{\prime 3}}-\frac{9}{8}\left(\frac{f^{\prime \prime}}{f^{\prime}}\right)\right)^{4}\right) f_{4}+ \\
& \left.+\frac{1}{4}\left(f^{\mathrm{IV}}-\frac{5}{2} \frac{f^{\prime \prime \prime} f^{\prime \prime}}{f^{\prime}}+\frac{5}{4} \frac{f^{\prime \prime 3}}{f^{\prime 2}}\right) f_{3}+\frac{3}{4}\left(f^{\prime \prime \prime} f^{\prime}-\frac{1}{2} f^{\prime \prime 2}\right) f_{2}+\frac{3}{2} f^{\prime \prime} f^{\prime 2} f_{1}\right\} .
\end{aligned}
$$

В дифференциальном операторе (12) частные производные функции $f$ по $y$ зависят от $\left(y_{a}, t_{a}\right)$, а функции $f_{i} \equiv f_{i}\left(f\left(y_{a}, t_{a}\right), t_{a}\right)$. Член с частной производной по $s$ вычисляется следуюшим образом: сначала берется $\frac{\partial}{\partial s} \ln f^{\prime 2}(y(s), \varphi(s))$ (производная по второму аргументу), где $\varphi(s)$ - решение уравнения (8), а затем делается переход $s=s_{a}$. После этого полученное выражение будет зависеть от $\left(y_{a}, t_{a}\right)$. Потенциал $V$ в формуле (12) имеет такую же зависимость от аргументов, как и функции $f_{i}$.

Полученный в результате преобразований оператор (12) обладает одной интересной особенностью. Коэффициенты оператора, пропорциональные $f_{4}$, вычисляются по вполне определенным правилам из коэффищиента, стояшего перед производной второго порядка. В частном случае, когда $f_{4}=1, f_{3}=f_{2}=f_{1}=V=0, f \equiv f(y)$, оператор (12) (без члена, пропорционального $E$ ) совпадает с конформно-ковариантным оператором, определенным на двумерной римановой поверхности. Такие операторы носят название операторов Бола $[10,11]$. Операторы Бола четвертого порядка имеют следующую структуру:

$$
L_{4}=\partial^{4}+5 R \partial^{2}+5(\partial R) \partial+\frac{3}{2}\left[\left(\partial^{2} R\right)+\frac{3}{2} R^{2}\right],
$$

где $R$ есть коэффициент проективной связности на римановой поверхности. В нашем случае объектом проективной связности является производная Шварца. Для коэффициентов оператора Бола произвольного порядка существует рекуррентная процедура, при которой все коэффициенты также выводятся из проективной связности.

Заметим, что операторы Бола появляются в уравнениях для тождеств Уорда в двумерных конформных моделях, а также в скобках Пуассона, порождающих классические алгебры Вирасоро и $W_{n}$-алгебры $[11]$. 


\section{5. ЗАКЛЮЧЕНИЕ}

Результаты, полученные в данной статье, а также в $[1,2]$, свидетельствуют о том, что предложенный метод исследования континуальных интегралов может быть распространен и на континуальные интегралы для дифференциальных уравнений высших порядков.

Обнаруженные геометрические свойства производящего оператора для преобразованной полугруппы свидетельствуют о том, что, по-видимому, сушествуют и другие, не связанные со случайными процессами по квазимерам способы доказательства полученных в работе формул.

Кроме того, такие геометрические свойства значительно расширяют область применения найденных формул, помимо их использования для точного вычисления континуальных интегралов и изучения влияния релятивистских поправок при квантовом движении.

Автор благодарен академику А. А. Логунову за оказанную поддержку.

\section{Список литературы}

[1] С. Н. Сторчак. ТМФ. 1992. Т. 93. С. 17-31.

[2] С. Н. Сторчак. ТМФ. 1992. Т. 93. С. 375-385.

[3] В. Ю. Крилов. ДАН СССР. 1960. Т. 132. С. 1254-1257.

[4] K. J. Hochberg. Ann. Prob. 1978. V. 6. P. 433-458.

[5] M. Motoo. An analogue to the stochastic integral for $\partial / \partial t=-\Delta^{4}$. In: Stoch. Analysis and Application, Adv. Prob. Related Topics. V. 7. Ed. M. Pinsky. N. Y.: Marcel Dekker, 1984.

[6] K. Nishioka. Japan. J. Math. 1985. V. 11. P. 59-102; J. Math. Soc. Japan. 1978. V. 39. P. 209-231.

[7] F. Steiner. Phys. Lett. A. 1984. V. 106. P. 356-362; C. Grosche, F. Steiner. Table of Feynmann Path Integrals. In: Springer Tracts in Modern Physics. Berlin: Springer-Verlag, 1995.

[8] И. И. Гихман, А. В. Скороход. Стохастические дифференциальные уравнения. Киев: Наукова думка, 1968

[9] S. N. Storchak. Phys. Lett. A. 1989. V. 135. P. 77-85.

[10] G. Bol. Abhandl. Math. Sem. Hamburger Univ. 1949. V. 16. P. 1; B. Gustafsson, J. Peetre. Nagoya Math. J. 1989. V. 116. P. 63.

[11] F. Gieres. Conformally covariant operators on Riemann surfaces (with application to conformal and integrable models). Preprint CERN-TH. 5985/91, MPI-Ph/91-39, June 1991.

Поступила в редакцию 26.VII.1996 г., после доработки 16.I.1997 г.

\section{S. N. Storchak \\ REONOMIC HOMOGENIOUS CONTACT TRANSFORMATIONS AND PATH REPARAMETRIZATION IN PATH INTEGRALS OVER QUASI-MEASURES}

In path integrals over quasi-measures for linear parabolic fourth order differential equations with the explicit dependence of coefficients on time the reonomic homogeneous contact transformations and path reparametrizations are considered. An integral relation between Green's functions for the fourth order differential equations being in correspondence is found. Differential operator of the transformed equation coincides in the particular case with the conformally covariant Bol operator, that is well-known in exactly solvable and conformal models. 